\title{
Exploring RSA Ultimate Accuracy by Using Computer Synthetic Images
}

\author{
Xunhua Yuan ${ }^{1,2}$, Terry M. Peters ${ }^{1}$, Robert B. Bourne ${ }^{2}$, and David W. Holdsworth ${ }^{1}$ \\ ${ }^{1}$ Imaging Research, Robarts Research Institute, P.O. Box 5015, 100 Perth Drive \\ London Ontario, Canada, N6A 5K8 \\ \{xyuan, dholdswo, tpeters\} @imaging.robarts.ca \\ ${ }^{2}$ Orthopaedics Division, London Health Sciences Centre, University Campus, \\ 339 Windermere Road, London, Ontario, Canada, N6A 5A5 \\ Robert. Bourne@lhsc.on.ca
}

\begin{abstract}
In this study, the ultimate accuracy of an RadioStereometric Analysis system was explored by computer synthetic images. Comparing computer synthetic images with joint phantom images, the correlation between RSA accuracy and image noise was revealed. Our study indicates that RSA has ultimate accuracies of $0( \pm 1) \mu \mathrm{m}, 0( \pm 1) \mu \mathrm{m}$ and $0( \pm 4) \mu \mathrm{m}$ along the X-, Y- and Zaxis, respectively, far more accurate than the phantom images with accuracies of $5( \pm 38) \mu \mathrm{m}, 6( \pm 44) \mu \mathrm{m}$ and $13( \pm 76) \mu \mathrm{m}$.
\end{abstract}

\section{Introduction}

RadioStereometric Analysis (RSA) [1] is a highly accurate radiological imaging technique that has been used in orthopaedic clinical research for many years [1, 2]. RSA technique includes the implantation of radiopaque tantalum markers into the skeleton and onto an implant to serve as landmarks, the use of stereo-radiographs to determine their three-dimensional positions, and the use of rigid body theory to calculate the relative motion between different bones or between implants and bones. Currently, RSA represents the gold standard for measuring implant micromotion from diagnostic medical radiographs.

Improved long-term survival of implanted prostheses is a challenge in orthopaedic surgery. Several studies have revealed that early implant micromotion is a prognostic indicator of long term survival [3]. Using RSA to detect early micromotion directly depends on the accuracy of the system. A highly accurate RSA system not only guarantees that small motions will be detectable, but also promises motion will be detected earlier. This is particularly important in clinical micromotion detection, especially when the micromotion approaches RSA accuracy limits.

Previously, the accuracy of RSA has been reported controversially, ranging from $0.03^{\circ}$ to $0.6^{\circ}$ for rotation and from $0.01 \mathrm{~mm}$ to $0.25 \mathrm{~mm}$ for translation [1-4]. The controversy has resulted from different factors such as different RSA set-ups, different tantalum bead settings and different image measurement software [5, 6]. In order to minimize the influence of these factors and enable accuracy evaluation comparable in different situations, a joint phantom was introduced [7] by mimicking anatomic geometry and joint movement. However, from the mechanical point of view, any 
physical models are subject to mechanical errors, therefore are limited in the accuracy with which measurements may be made. This becomes an obstacle in assessing micromotion that is of the same order as the phantom accuracy.

Improving RSA accuracy is one of challenges in using this technique. Many approaches have been performed, from the investigation of the influence of marker numbers, the distance effect, the calibration cage design and the patient spatial position $[1,3,5,6]$, to the upgrade of RSA from conventional film to digital image $[8,9]$. Although these studies uncovered some inherent features of the RSA system, the ultimate limits of accuracy remain unclear. It is expected that reducing image noise will increase the accuracy of RSA, thus, a study that eliminates image noise will help us to understand how much improvement in accuracies could be achieved.

We believe that the RSA technique is capable of much higher accuracy than previously reports. In order to evaluate the fundamental limits of the RSA technique, we propose a numerical simulation whose results can be compared with those obtained from real images.

\section{Methods}

\subsection{RSA Procedure}

The RSA procedure involves four steps. The first is the surgical insertion of tantalum markers with the diameter of $0.8 \mathrm{~mm}$ or $1.0 \mathrm{~mm}$. The markers are either inserted into skeletal segments or onto implant segments, with at least three markers on each in order to construct rigid body models. After surgery, patients undergo several followup radiographic examinations. Each radiographic examination requires two X-ray tubes exposed simultaneously from two different projections, and a special calibration cage comprised of several embedded tantalum markers. The cage is used to define a three-dimensional coordinate system, and to reconstruct the position of the two X-ray foci. Following radiographic examination, the cage and patient markers are identified on both radiographs and are measured by a special software. Based on these twodimensional measurement results, in the end, three-dimensional marker positions are reconstructed and the relative movement between different segments is calculated according to rigid body theory.

\subsection{Principle of Computer Synthetic Image Generation}

In order to evaluate RSA accuracy independent of image noise, a computer synthetic image was introduced. The generation of computer synthetic images was based on the perspective projection of a sphere. Mathematically, when the relative positions of a sphere and an X-ray focus with respect to a detector plane are known, the perspective elliptic shadow of the sphere on the plane can be uniquely determined (Fig. 1) and described as following.

$$
\mathrm{E}(x, y)=\mathrm{F}\left(\mathbf{P}_{0}\left(x_{0}, y_{0}, z_{0}\right), \mathbf{P}(x, y, z), R\right)
$$

where F contains three variables: $\mathbf{P}_{0}$ represents the spatial position of an X-ray focus, $\mathbf{P}$ the position of a sphere and $R$ the radius of the sphere. 


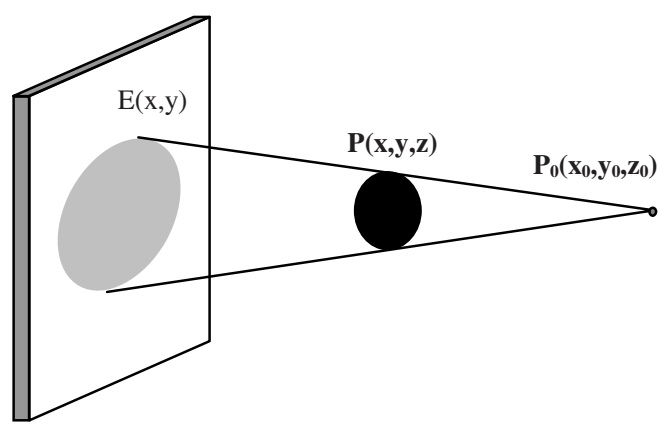

Fig. 1. The graph of sphere perspective projection

We applied this principle to a femoral head, an acetabular cup, all calibration cage markers and patient beads, and calculated their projective shadows as described by a digital image format. This resulted in a computer synthetic image corresponding to a hip joint (Fig. 2a). An in-house Matlab (The Mathworks Inc, Natick, Massachusetts, USA) program was developed to generate this image. Illustration

To generate a computer synthetic image corresponding to a phantom image, 3D positions of all beads, the femoral head and the acetabular cup, as well as the positions of the $\mathrm{x}$-ray foci, were extracted from the initial phantom image measurement with UmRSA (RSA Biomedical Innovations AB, Umeå, Sweden) (Fig. 2b). Once the initial computer synthetic image was generated, 19 subsequent synthetic images representing the head movements relative to the acetabular cup (in a manner similar to phantom movements driven by the 3D positioning stage used in a phantom experiment) were computer generated from the initial position. Each image corresponds to one of the phantom images with the same image size, resolution, and femoral head movement (Fig. 2a, b).

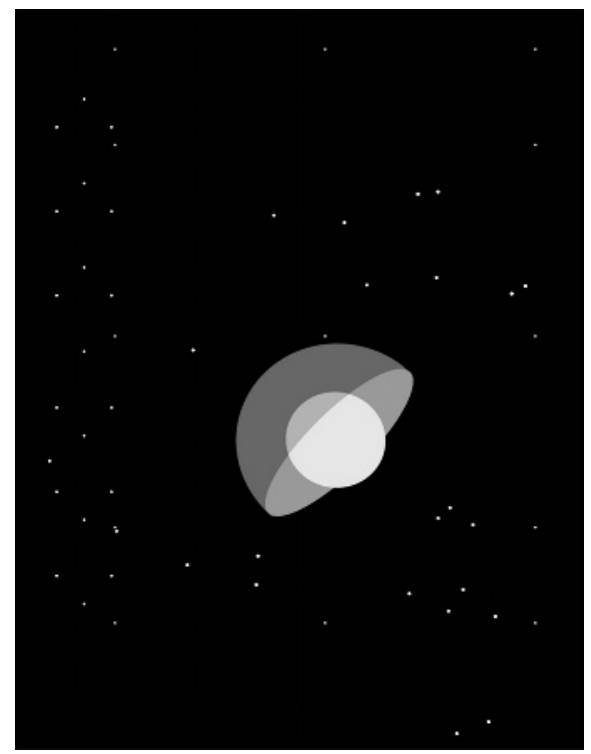

Fig. 2a. Computer synthetic image

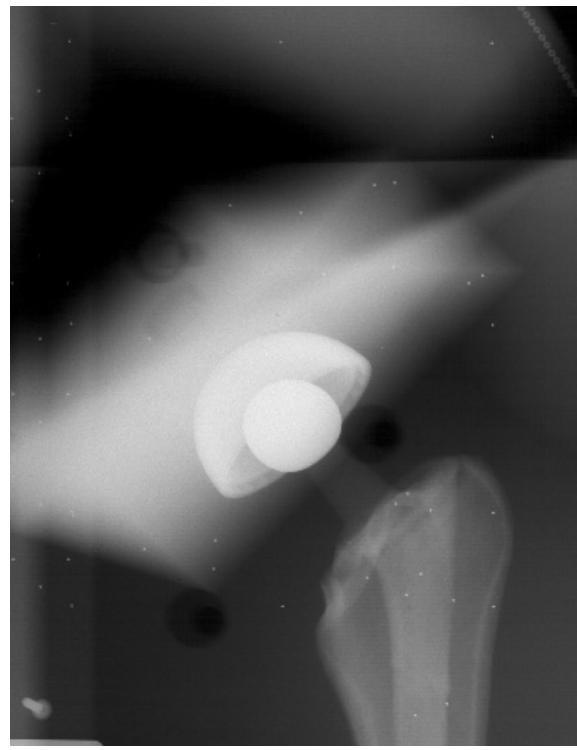

Fig. 2b. Hip joint phantom image 


\subsection{Phantom Construction and Examination}

A total hip joint phantom was constructed to simulate precise joint movements. The phantom consists of two portions (Fig. 3), a fixed acetabular cup and a movable femur.

A radiotranslucent polymethyl-methacrylate (PMMA) mounting block was used to mimic the radiodensity of soft tissue around the acetabular cup and was milled out to fit the cup. A $58 \mathrm{~mm}$ diameter titanium hemispherical acetabular standard Reflection ${ }^{\circledR}$ shell (Smith and Nephew Inc, Memphis, Tennessee) was fixed to the mounting block. A $28 \mathrm{~mm}$ diameter cobalt-chrome femoral head was attached to a polymer femoral stem that was cemented into a sawbone femur (Pacific Research Labs. Vashon Island,

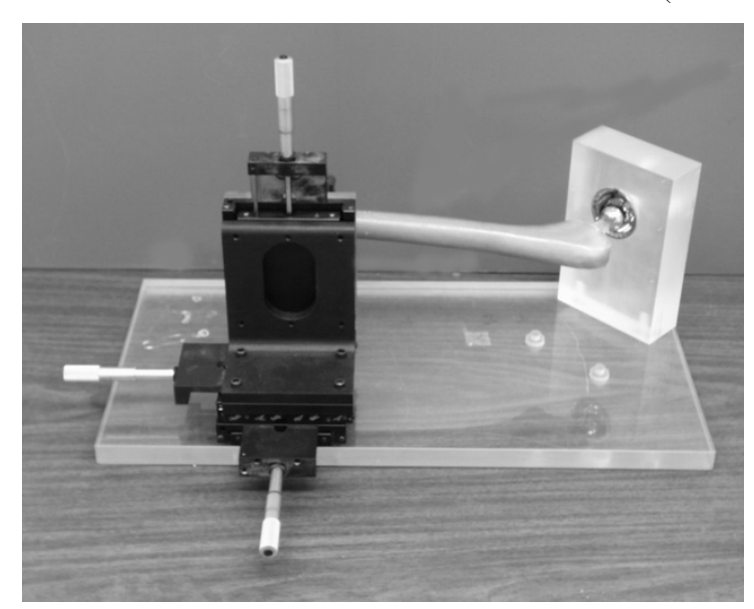

Fig. 3. A hip joint phantom

Washington, USA). The head-stem-bone composite was rigidly attached to a 3D positioning stage (Parker Hannifin Corporation, Harrison, PA, USA), with a resolution of $1 \mu \mathrm{m}$. In order to reproduce the RSA technique, nine evenly distributed tantalum beads were placed within the PMMA mounting block and another nine beads were inserted into the sawbone femur; three beads were placed at the upper femur, four beads around the middle and two near the tip of the stem.

The X-ray examination was undertaken by placing the phantom on a standard patient examination table. An Umeå uniplanar calibration cage (RSA Biomedical Innovations $\mathrm{AB}$, Umeå, Sweden) was placed under the table, allowing a standard ceiling mounted and a portable $\mathrm{x}$-ray unit to be used as the two required beam sources, separated by about 40 degrees and $140 \mathrm{~cm}$ above the calibration cage. Two standard $35 \times$ $43 \mathrm{~cm}$ X-ray film cassettes were placed under the calibration cage and radiographs of the phantom were taken initially at zero motion, followed by the movement of $50 \mu \mathrm{m}$, $100 \mu \mathrm{m}, 150 \mu \mathrm{m}, 200 \mu \mathrm{m}, 500 \mu \mathrm{m}$ and $1000 \mu \mathrm{m}$ along each plane for the sequence of $\mathrm{X}, \mathrm{Y}$ and $\mathrm{Z}$ planes. In total, 19 pairs of radiographs were produced. The processed $\mathrm{X}$ ray films were scanned into digital images using an UMAX Powerlook scanner (UMAX Technologies, Inc, Dallas, Texas, USA) with a resolution of 300 DPI at 8-bit grey scale level.

\subsection{RSA Calculation and Statistical Analysis}

Computer synthetic images and phantom images were measured using UmRSA Digital Measure ${ }^{\circledast}$ version 2.2.1 (RSA Biomedical Innovations AB, Umeå, Sweden), then motion was calculated by UmRSA version 4.1 (RSA Biomedical Innovations $\mathrm{AB}$, Umeå, Sweden). Accuracies were determined from the differences between RSA 
outcomes and true input movements for all three planes (X, Y and Z), individually. The magnitude of the accuracies were expressed by the mean plus/minus the standard deviation of these differences.

Linear regression analysis was used to determine the relationship between numerical input movements and RSA outcomes for computer synthetic images, and between phantom input increments and RSA outcomes for phantom images. Equality of means was tested for X-, Y- and Z-axis, respectively, using independent sample t-tests between the computer synthetic images and the phantom images. A significant difference between two groups of means was defined at the 5\% significance level ( $\mathrm{p}<$ 0.05). Statistical calculations were performed using the SPSS program (SPSS Inc., Chicago, Illinois, USA).

\section{Results}

The RSA system demonstrated extremely high accuracies with $0( \pm 1) \mu \mathrm{m}, 0( \pm 1) \mu \mathrm{m}$ and $0( \pm$ 4) $\mu \mathrm{m}$ for synthetic images along the $\mathrm{X}-$, $\mathrm{Y}$ - and Z-axis, respectively. The accuracies for the phantom images were 5

$( \pm 38) \mu \mathrm{m}, 6( \pm 44) \mu \mathrm{m}$ and $13( \pm 76) \mu \mathrm{m}$ along the $\mathrm{X}-, \mathrm{Y}-$ and Z-axis, respectively (Table 1).
Table 1. Accuracies of computer synthethic images and phantom images represented by mean \pm standard deviation $(\mu \mathrm{m})$

\begin{tabular}{lccc}
\hline & $\mathrm{X}$ & $\mathrm{Y}$ & $\mathrm{Z}$ \\
\cline { 1 - 3 } Computer Synthetic Images & $0 \pm 1$ & $0 \pm 1$ & $0 \pm 4$ \\
Phantom Imags & $5 \pm 38$ & $6 \pm 44$ & $13 \pm 76$
\end{tabular}

Phantom Imags $\quad 5 \pm 38 \quad 6 \pm 44 \quad 13 \pm 76$

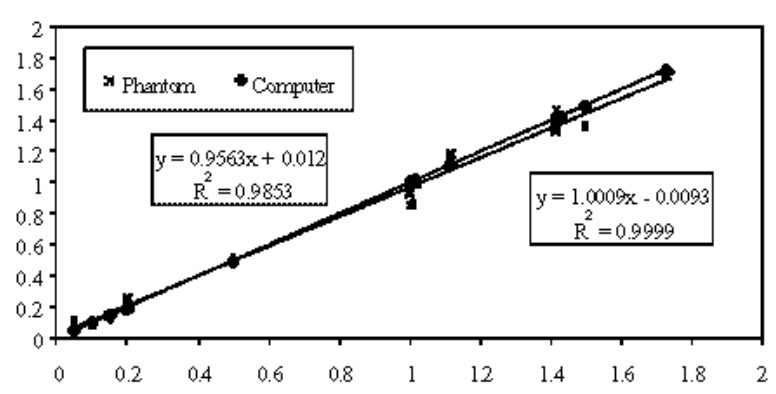

Fig. 4. Illustration of linear regression

Linear regression analysis showed that outcomes from both computer synthetic images and phantom images have statistically significant correlations with the true movements (Fig. 4). The computer synthetic images showed a stronger trend with an extremely high $\mathrm{R}^{2}$ value $\left(\mathrm{R}^{2}=0.9999\right)$ compared to phantom images. Comparing the computer synthetic images directly with the phantom images, independent sample ttests confirmed that a significant difference exists between the two image groups with significant level $(\mathrm{p}<0.001)$. 


\section{Discussions}

\subsection{Accuracy Evaluation}

RSA has been used to observe implant micromotion for many years. However, accuracy and precision of RSA has been defined in many ways. In "in vivo" clinical RSA studies, double exposure examinations have been used in the evaluation of either accuracy $[10,11]$ or precision [8] based on the assumption of zero movement between exposures. In "in vitro" studies, accuracy has been defined as the similarity of measurements to references, while precision was the agreement between test results [12]. The magnitude of accuracy and precision were expressed as either median [11], or mean with standard deviation [8], or 95\% prediction interval [12]. In this study, the American Society for Testing and Material Standard (ASTM E 177-90a) definitions were used. Accuracy was defined as the image measurement versus input increment and the magnitude of the accuracy was expressed by the mean plus/minus the standard deviation (SD) of the calculated sets.

Correct evaluation of RSA accuracy is compromised clinically by the inability to obtain true motion data [5, 7]. The analysis of RSA accuracy has to be performed non-clinically, either by a physical model or by a numerical model, in order to obtain true movements. In this study, a hip joint phantom was constructed as a physical model and the computer synthetic image was proposed as a numerical model. These models exhibit two different levels of image noise, thus providing insight into the accuracy of RSA with respect to the image noise, which facilitates the interpretation of the actual patient-related image measurement.

Any object movement in a 3D space has six degrees of freedom (DOF) in which three are rotation and the other three are translation around/along the $\mathrm{X}-, \mathrm{Y}-$ and $\mathrm{Z}$ axis, respectively. In this study, accuracy analysis was only performed for translation, not for rotation. Based on the rigid body theory, the translation and the rotation are intrinsically dependent, and higher translation accuracy guarantees higher rotational accuracy and vice versa $[8,11]$. Therefore in this study, the accuracy of the rotational analysis was not performed.

\subsection{Repeatability and Reproducibility of RSA}

Once RSA measurements are deemed to be accurate, the precision of the RSA system must be considered. According to the definition of ASTM, precision can be expressed by two forms: repeatability and reproducibility. Simply, repeatability is an evaluation of results by the same operator using the same equipment within short intervals of time, while reproducibility is defined as different operators using different equipment.

The repeatability of RSA has been explored by many studies. Using double exposures from patient examinations, results showed the repeatability of RSA is less than $0.2 \mathrm{~mm}$ for hip [8] and less than $0.23 \mathrm{~mm}$ for knee [11], while from knee phantom examinations, the repeatability of $0.13 \mathrm{~mm}$ was reported, an increase of about $50 \%$ from patient knee examinations. A potential problem with these results is that micromotion could take place in the interval between double exposures, thereby leading to an under-estimation of RSA repeatability. The use of a phantom model or synthetic images removes this limitation. 
The repeatability of radiograph measurements (upon which RSA analysis depends) has been evaluated previously. By double scanning radiographs without moving them between the two scans, it has been shown that the repeatability is less than $0.081 \mathrm{~mm}$ [13]. With double measurements of the same radiographs at different times, our previous study demonstrated extremely high measurement repeatability with errors less than $0.013 \mathrm{~mm}$ [5]. Such performance relates to the methodology of bead measurement. With UmRSA ${ }^{\circledR}$, the bead detection is regionally applied. Initial bead positions are approximately identified by an observer with a mouse and subsequently refined in a region of interest (ROI), $35 \times 35$ pixel window size surrounding the initial position [8]. The refinement is based on the optimal fitting within the ROI to a marker model and therefore, the final refined measurement depends on the gray-scale level of the pixels in the ROI, rather than the initial bead position, making the radiograph measurements nearly operator independent.

\subsection{Computer Synthetic Images vs. Phantom Images}

Historically, the evaluation of RSA accuracy began with the design of physical models $[14,15]$. The initial models had fairly simple structures, using either wooden rods or plexiglass tubes. As the models lack anatomic structures, they are unable to mimic real skeletal movement, limiting the accuracy analysis to only zero increment movement. More recently by mimicking the dominant skeletal structures, joint phantoms were introduced $[5,7]$, enabling the joint movement to be precisely simulated. However, joint phantoms did not consider the influence of soft tissue, and were unable to reproduce features related to it, such as image noise (which is one of the key factors affecting RSA accuracy).

Until recently, there were few studies to quantitatively analyze image noise related to RSA accuracy, due to the complexity of different types of noise, the propagation of which are difficult to analyze in clinical investigations. In this paper, we proposed an approach to circumvent this problem by comparing phantom images with computer synthetic images. Qualitatively, the computer synthetic images shared the same features and appearance as the phantom images in terms of motion increments, dimensions and spatial positions of the head, cup and tantalum markers, and image size and resolution, differing from the phantom images only in image noise. Analyzing both types of images, the improvement in RSA accuracy in the absence of image noise could be evaluated.

Computer synthetic images can be an essential tool in evaluating different RSA systems, since these studies can be carried out in the absence of image noise and without being limited to specific X-ray imaging systems. They are also useful in designing new calibration cages by pre-demonstrating the effects of bead location, bead numbers and object spatial positions. In addition, computer synthetic images can provide a theoretical framework for testing new kinematic and dynamic analysis approaches. 
Acknowledgements. This study was financially supported by Smith \& Nephew Canada.

\section{References}

1. G. Selvik: Roentgen stereophotogrammetry. A method for the study of the kinematics of the skeletal system. Acta Orthop Scand Suppl 232 (1989) 1-51

2. J. Kärrholm, P. Herberts, P. Hultmark, H. Malchau: Radiostereometry of hip prostheses. Review of methodology and clinical results. Clin Orthop 344 (1997) 94-110

3. L. Ryd: Micromotion in knee arthroplasty. A roentgen stereophotogrammetric analysis of tibial component fixation. Acta Orthop Scand Suppl 220 (1986) 1-80

4. J. Kärrholm: Roentgen stereophotogrammetry. Review of orthopedic applications. Acta Orthop Scand 60 (1989) 491-503

5. X. Yuan, J. Patel, R.W. McCalden, S.J. MacDonald: Accuracy and Precision of Digital RSA Related to Image Quality. J of Arthroplasty Submitted (2002)

6. X. Yuan, L. Ryd and L. Blankevoort: Error propagation for relative motion determined from marker positions. J Biomech 30 (1997) 989-992

7. C.R. Bragdon, D. Estok, H. Malchau, J. Kärrholm: In vitro Comparison of Two Different Radiostereometric Analysis (RSA) Methods in the Determination of Femoral Head Penetration into Polyethylene in a Total Hip Replacement Phantom while using Digital Radiography. (2002)

8. N. Börlin, T. Thien and J. Kärrholm: The precision of radiostereometric measurements. Manual vs. digital measurements. J Biomech 35 (2002) 69-79

9. H.A. Vrooman, E.R. Valstar, G.J. Brand, D.R. Admiraal: Fast and accurate automated measurements in digitized stereophotogrammetric radiographs. J Biomech 31 (1998) 491-498

10. H. Franzén and B. Mjoberg: Wear and loosening of the hip prosthesis. A roentgen stereophotogrammetric 3-year study of 14 cases. Acta Orthop Scand 61 (1990) 499-501

11. E.R. Valstar, H.A. Vrooman, S. Toksvig-Larsen, L. Ryd: Digital automated RSA compared to manually operated RSA. J Biomech 33 (2000) 1593-1599

12. I. Önsten, A.S. Carlsson and J. Besjakov: Wear in uncemented porous and cemented polyethylene sockets: a randomised, radiostereometric study. J Bone Joint Surg Br 80 (1998) 345-350

13. S.E. Østgaard, L. Gottlieb, S. Toksvig-Larsen, A. Lebech: Roentgen stereophotogrammetric analysis using computer-based image-analysis. J Biomech 30 (1997) 993-995

14. A. Lundberg, C. Bylund, G. Selvik and I.G. Winson: Accuracy of Roentgen Stereophotogrammetric Analysis in Joint Kinematics. Eur J Exp Musculoskel Res 1 (1992) 213-216

15. S. Resch, L. Ryd, A. Stenstrom and X. Yuan: Measurement of the forefoot with roentgen stereophotogrammetry in hallux valgus surgery. Foot Ankle Int 16 (1995) 271-6 